\title{
Letter to the Editor:'Euthyroid sick syndrome as an early surrogate marker of poor outcome in mild SARS-CoV-2 disease'-prognostic significance of non-thyroidal illness syndrome across the whole spectrum of COVID-19 severity
}

\author{
David Tak Wai Lui ${ }^{1} \cdot$ Chi Ho Lee ${ }^{1} \cdot$ Karen Siu Ling Lam ${ }^{1}$
}

Received: 11 December 2021 / Accepted: 21 December 2021 / Published online: 13 January 2022

(C) Italian Society of Endocrinology (SIE) 2022

\section{Dear Editor,}

We read with great interest the recent publication by Sparano et al. on the prognostic significance of fT3 in COVID-19 [1], in which consecutive hospitalized mild COVID patients had TSH, fT4 and fT3 evaluated on admission. Remarkably, abnormal thyroid function (defined as any abnormality in TSH, fT4 and fT3) occurred in 70\%, and non-thyroidal illness syndrome (NTIS) in 57\% of the cohort. In contrast, with a very similar study protocol, we previously reported lower rates of abnormal thyroid function at $16.9 \%$ and NTIS at $7.4 \%$ in our cohort with predominantly mild-to-moderate COVID-19 patients in Hong Kong (75\% mild) [2].

The apparent paradox demonstrated in Sparano's study that mild patients showed a much higher rate of NTIS than the mild-to-moderate patients in our study could be possibly explained by the differences in clinical severity scoring systems adopted by the two studies. Sparano's study included patients with mild severity defined by the World Health Organization's (WHO) Ordinal Scale for Clinical Improvement, meaning hospitalized patients without oxygen requirement (category 3 ), and those requiring oxygen via mask/nasal prongs (category 4). Radiological manifestation of pneumonia is not a consideration in this classification. In contrast, patients in our study were classified according to the Chinese National Health Commission (NHC) guideline, which is similar to the one proposed by the National Institutes of Health, with higher resolution: mild-no manifestation of pneumonia; moderate-evidence of pneumonia but without desaturation; severe-desaturation requiring

Karen Siu Ling Lam

ksllam@hku.hk

1 Department of Medicine, The University of Hong Kong, Hong Kong, China supplemental oxygen. Therefore, it will be interesting to know the proportion of patients having abnormal chest X-ray (from mild involvement onwards) or requiring oxygen on admission in Sparano's study, so that the two cohorts can be compared, using the same severity definition, and evaluate whether possible geographical variations or differences exist across the period of pandemic. Indeed, Sparano's study may have included patients with a more severe disease spectrum (mean $\mathrm{PO}_{2} / \mathrm{FiO}_{2}$ at admission was $<300 \mathrm{mmHg}$ - which would be classified as severe according to NHC guideline), thus associated with a higher rate of NTIS. On the other hand, it is important to note that the public health ordinance in Hong Kong required all patients who were tested positive for COVID-19 be admitted to the hospital, including those detected on contact tracing and Universal Community Testing Programme, regardless of symptoms [2]. Although our study evaluated hospitalized patients, our cohort is likely representative of the general population at large. Therefore, the results of ours and Sparano's study might in fact complement each other, with our study demonstrating the prognostic role of NTIS in the COVID-19 patients in the community at large, while Sparano's study further demonstrating the prognostic role of NTIS in non-intensive care hospitalized patients.

Putting all the existing studies together, NTIS has been shown to carry prognostic significance in COVID-19 across the whole spectrum of disease severity [3]. Looking ahead, it may be helpful to harmonize the severity classification criteria in future systematic reviews or meta-analyses of NTIS in COVID-19 for better understanding of thyroid dysfunction in COVID-19. 


\section{Declarations}

Conflict of interest On behalf of all authors, the corresponding author states that there is no conflict of interest.

Ethical approval This article does not contain any studies with human participants or animals performed by any of the authors.

Informed consent No informed consent.

\section{References}

1. Sparano C, Zago E, Morettini A et al (2021) Euthyroid sick syndrome as an early surrogate marker of poor outcome in mild
SARS-CoV-2 disease. J Endocrinol Invest. https://doi.org/10. 1007/s40618-021-01714-1

2. Lui DTW, Lee CH, Chow WS et al (2021) Role of non-thyroidal illness syndrome in predicting adverse outcomes in COVID-19 patients predominantly of mild-to-moderate severity. Clin Endocrinol (Oxf) 95(3):469-477. https://doi.org/10.1111/cen.14476

3. Llamas M, Garo ML, Giovanella L (2021) Low free-T3 serum levels and prognosis of COVID-19: systematic review and metaanalysis. Clin Chem Lab Med 59(12):1906-1913. https://doi.org/ 10.1515/cclm-2021-0805

Publisher's Note Springer Nature remains neutral with regard to jurisdictional claims in published maps and institutional affiliations. 